\title{
Phototransduction in ganglion-cell photoreceptors
}

\author{
David M. Berson
}

Received: 24 December 2006 / Accepted: 13 February 2007 / Published online: 10 March 2007

(C) Springer-Verlag 2007

\begin{abstract}
A third class of photoreceptors has recently been identified in the mammalian retina. They are a rare cell type within the class of ganglion cells, which are the output cells of the retina. These intrinsically photosensitive retinal ganglion cells support a variety of physiological responses to daylight, including synchronization of circadian rhythms, modulation of melatonin release, and regulation of pupil size. The goal of this review is to summarize what is currently known concerning the cellular and biochemical basis of phototransduction in these cells. I summarize the overwhelming evidence that melanopsin serves as the photopigment in these cells and review the emerging evidence that the downstream signaling cascade, including the light-gated channel, might resemble those found in rhabdomeric invertebrate photoreceptors.
\end{abstract}

Keywords Melanopsin · ipRGC · Photoreceptor ·

Rhabdomeric $\cdot$ Retina $\cdot$ Ganglion cell .

Suprachiasmatic nucleus · TRPC

Within the past decade, the existence of a previously unknown class of photoreceptor in the mammalian retina has come to light. These directly photosensitive neurons differ radically in form and function from the well-known rod and cone photoreceptors. They are a rare variety of retinal ganglion cells (RGCs), the class of retinal neurons sending axons through the optic nerve to synapse in the brain. For at least the prior century, it had gone without question that ganglion cells were entirely dependent on polysynaptic influences from rods and cones for their

D. M. Berson $(\bowtie)$

Department of Neuroscience, Brown University,

P.O. Box G-L4, Providence, RI 02912, USA

e-mail: David_Berson@brown.edu responsiveness to light (indeed, even today there is no reason to doubt this for most ganglion cells). However, as the new millennium dawned, this dogma was being challenged by behavioral studies showing the persistence of certain reflex responses to light in photoreceptor degenerate animals, e.g., $[14,15,35,36]$, and the presence of a possible photopigment, melanopsin, in a rare population of ganglion cells [18, 19, 21, 49, 50]. In short order, it became clear that these cells possessed a capacity for autonomous phototransduction [5]. These intrinsically photosensitive RGCs (ipRGCs) are now recognized as playing key roles in synchronizing circadian rhythms to the day-night cycle, mediating the pupillary response to light, and modulation of melatonin release by the pineal gland. Many aspects of this rapidly developing story have been thoroughly summarized elsewhere $[4,6,14,16,31,44,54,61]$. The reader is referred to these reviews for background on the intellectual origins of the discovery of melanopsin and the ipRGCs, on their structure and physiology, and on their contribution to circadian photoentrainment and other nonimage-forming visual functions. The goal of the present review is to summarize the current state of knowledge concerning the nature of the transduction process in ipRGCs.

\section{Structural and functional properties of ipRGCs relevant to the phototransduction process}

The ipRGCs represent a tiny minority of the RGCs of mammalian retinas $(<1-2 \%)[9,21,51]$. Like other ganglion cells, ipRGCs extend dendritic processes into a synaptic lamina of the retina, the inner plexiform layer, and send a single axon through the optic nerve to terminate in the brain. The ipRGCs are distinguishable from other RGCs, however, by their selective expression of a novel 
opsin called melanopsin and of a peptide neuromodulator, pituitary adenylate cyclase activating polypeptide [19]. These cells also exhibit a distinctive pattern of central axonal projection, with major targets including nuclei coordinating circadian rhythms and mediating pupillary responses [21]. Their most notable functional idiosyncrasy, of course, is their ability to respond to light when totally isolated from any synaptic influence [5, 9, 60, 62]. It should be noted, however, that in addition to this direct sensitivity to light, ipRGCs receive excitatory and inhibitory synaptic inputs that permit rods and cones to modulate their electrical behavior [2, 46; Wong et al. unpublished observations].

The direct photoresponse of ipRGCs to light can be evoked not only by diffuse illumination but also by stimuli restricted either to the soma or to the dendrites [5], indicating that the phototransduction apparatus is widely dispersed through the cell. Illumination induces a conductance increase and membrane depolarization, which, if sufficiently large, can trigger repetitive regenerative action potentials. The responses are generally very sluggish, with a latency of several seconds from light onset to response peak when probed with stimulus intensities in the middle of the dynamic range. However, the intensity-latency relation is steeply inverse, with latencies ranging from several hundred milliseconds for saturating stimuli to $>1 \mathrm{~min}$ for near-threshold stimulation. Responses to prolonged light steps generally decay significantly from their initial peak, in part because of light adaptation [63], but the steady-state response is generally remarkably stable. This stability seems likely to be a key functional difference from rods and cones, although a systematic exploration of this difference has not yet been attempted. The ability of ipRGCs to signal the presence of steady illumination over many minutes is especially relevant in the context of the reflexive responses to ambient light to which they contribute. Like the ipRGCs themselves, these visual responses are remarkably tonic and/or exhibit unusually long integration times. Response recovery in ipRGCs, like response onset, is strikingly slow by comparison with rods and cones. Typically, several minutes in darkness are required for a photoactivated ipRGC to return to its prestimulus voltage $[5,60]$.

\section{Identity and properties of the ipRGC photopigment}

There is overwhelming evidence that melanopsin is the functional photopigment of ipRGCs. This opsin was first discovered in a screen for opsin-like genes in cDNA library from Xenopus dermal melanophores, a nonneural cell type that redistributes its pigment in direct response to light [49]. Orthologs of the gene were then identified in mammalian genomes, including those of mice, monkeys, and humans [50]. A striking feature of melanopsin is that its predicted amino acid sequence exhibits greater homology with the opsins of invertebrate rhabdomeric photoreceptors ("r-opsins") than with the opsins of vertebrate ciliary photoreceptors (“c-opsins") [1, 30, 49, 50, 57].

In mammals, melanopsin is apparently expressed almost exclusively within a rare population of RGCs $([9,19,22$, $50,51]$ but see [11] and [45]). These RGCs are the main source of retinal input to the suprachiasmatic nucleus of the hypothalamus, the brain's circadian pacemaker [18, 19, 21, 22]. Experiments combining recording, intracellular dye filling, and immunofluorescence established that melanopsinexpressing cells are directly photosensitive $[5,22]$. The spectral tuning of the ipRGC photoresponse adheres closely to the form expected for an opsin-based response [5]. Immunohistochemistry identified the presence of melanopsin protein throughout the dendritic arbor of ipRGCs as well as in the soma $[9,19,22,50,51]$. This distribution bolsters melanopsin's hypothesized status as the ipRGC photopigment, because, as already noted, both the dendrites and somata of ipRGCs have an independent capacity for phototransduction. In fact, melanopsin has been observed even in the proximal, intraretinal portion of ipRGC axons. This suggests that the axons, too, could be at least weakly photosensitive, although this has not been tested. Melanopsin appears localized almost entirely to the plasma membrane of ipRGCs [2]. There is no evidence for any membranous specializations in ipRGCs analogous to the disks of rod or cone outer segments or the microvilli of rhabdomeric photoreceptors. Those specializations serve to dramatically increase membrane surface area and thus the density of the photopigment. Thus, an incident photon is presumably much less likely to be captured by the melanopsin in an ipRGC than by the photopigments of rod or cone outer segments or ommatidial rhabdomeres. This is one likely contributor to the relatively low sensitivity of ipRGCs by comparison with rods and cones $[5,9]$.

Key support for the hypothesis that melanopsin is the ipRGC photopigment came from the observation that the direct photoresponse of these cells was abolished by targeted deletion of the melanopsin gene [37; see also 23, 42]. By itself, however, this finding did nothing to exclude an alternative model in which melanopsin served not as a photopigment but rather as a photoisomerase. According to this model, melanopsin might be essential for providing cis retinaldehyde chromophore to the actual ipRGC photopigment, which remained to be identified [3]. However, parallel studies provided evidence that melanopsin could form a functional photopigment, inasmuch as it mediated light-triggered G-protein activation in a biochemical assay [40]. Further, evidence soon emerged that heterologous expression of melanopsin conferred intrinsic photosensitiv- 
ity on each of three otherwise light-insensitive cell types $[38,41,52]$. Moreover, the action spectrum of the induced photoresponses closely matched that of the ipRGCs, peaking in the blue portion of the visible spectrum $(\sim 480 \mathrm{~nm}$; [41, 52]; see also [30]). This close spectral congruence has not been seen universally. Spectrophotometric observations on purified melanopsin initially suggested a peak absorption closer to $420 \mathrm{~nm}$ [40], and a similar value was obtained from electrophysiological measurements in one heterologous expression system [38]. More recent evidence suggests that if the composition of the extraction buffer is altered, purified melanopsin does indeed absorb maximally near $480 \mathrm{~nm}$ (Walker et al., personal communication; see also [30]). Thus, with the exception of the report of Melyan et al. [38], there is good agreement that melanopsin can account for the spectral behavior of ipRGC photoresponses. The question of whether the shorter wavelength absorbance maximum seen in some experimental settings occurs under physiological conditions in any native cellular environment is unresolved.

The identity of the melanopsin's retinaldehyde chromophore is unknown, although the opsin can form a functional photopigment when reconstituted with all-trans, 9-cis, 11cis, or 13-cis retinaldehyde ([17, 38, 40, 41, 52], and Qiu and Berson, unpublished observations). The various states through which the pigment passes during photoexcitation and pigment regeneration are also largely mysterious. However, clues may come from the invertebrate-like structure of melanopsin generally and the invertebrate-like identity of the residue thought to serve as the Schiff-base counterion in particular [49]. These suggest that melanopsin may form a bistable, photoreversing pigment. In such pigments, photon absorption and isomerization of cis to trans retinal generates a metarhodopsin species that is thermally stable. Rhabdomeric metarhodopsin can function as a photoisomerase, absorbing light to convert its retinal chromophore from trans to cis retinal and reverting to photoexcitable rhodopsin [24]. This is very different from the arrangement in rod and cone photopigments ("c-opsins"), in which the metarhodopsin is thermally unstable and quickly dissociates into free retinal and naked opsin apoprotein. Pigment regeneration requires multiple enzymatic steps, most of which are localized to the retinal pigment epithelium (RPE). Were melanopsin truly bistable, it could help to explain why ipRGCs retain photoresponsiveness for many hours in the absence of the RPE [5]. Indeed, photoreversal has been observed spectrophotometrically in melanopsin from amphioxus, a primitive chordate [30]. Several studies have shown that functional photopigment can be obtained by loading melanopsin with all-trans retinal $[17,38,41,52]$, presumably because the opsin can catalyze formation of cis retinal. Melyan et al. [38] also obtained evidence that exposure to relatively long wavelengths sensitized subsequent melanopsin-based photoresponses, presumably by driving a trans-to-cis isomerization of retinal chromophore and thus increasing the concentration of excitable melanopsin. We have recently observed electrophysiological evidence for photoreversal in ipRGCs themselves [Qiu and Berson, unpublished observations]. When a saturating light stimulus is extinguished, the light-evoked depolarization decays very gradually (over $>2 \mathrm{~min}$ ) toward prestimulus levels. This poststimulus persistence resembles the persistent depolarizing afterpotential (PDA) of rhabdomeric photoreceptors, a phenomenon attributable to a persistent pool of thermally stable metarhodopsin [24]. During the PDA in ipRGCs, exposure to yellow light $(560 \mathrm{~nm})$ drives the ipRGC membrane potential back toward baseline, just as it does in rhabdomeric photoreceptors, strongly suggesting that melanopsin is bistable in mammalian ipRGCs. There is at least one observation, however, in apparent conflict with the view that intrinsic photoisomerization of melanopsin's chromophore is primarily responsible for pigment regeneration in ipRGCs. Fu et al. [17] reported that knocking out RPE65, an essential retinal isomerohydrolase of the RPE, reduced ipRGC sensitivity as assessed indirectly from pupillary responses. This result appeared to suggest that melanopsin depended at least in part on an extrinsic enzymatic retinoid cascade for its normal function. However, more recent evidence indicates that this effect is secondary to the devastating effects of the knockout on rod and cone function ([59]; see also [13, 34]). In mice with degenerative loss of rods and cones, neither RPE65 deletion nor acute pharmacological disruption of the retinoid cycle has any significant impact on ipRGC sensitivity [59]. These results do not entirely exclude a retinoid cycle for ipRGCs localized within the inner retina and involving proteins other than melanopsin itself, but at present, there seems no strong evidence favoring such a mechanism. Nonetheless, it is worth noting that the poststimulus depolarization of ipRGCs decays spontaneously (if sluggishly) in darkness, after which ipRGC sensitivity returns to prestimulus levels ([5, 64]; Qiu and Berson, unpublished observations). These results suggest that photoexcited melanopsin is not indefinitely thermostable and that there may be light-independent pathways capable of regenerating photoexcitable melanopsin.

\section{Phototransduction cascade: the role of $G$ proteins}

By comparison with the widespread consensus about the identity of the photopigment in ipRGCs, the current state of understanding of the signaling cascade linking melanopsin to the light-gated conductance can only be described as sketchy. Opsins are heptihelical G-protein coupled receptors (GPCRs), and photoisomerization triggers heterotrimeric G-protein activation in all well-characterized 
bilaterian phototransduction cascades. There is some evidence that this is true in ipRGCs [63], although the evidence is not decisive. Nonetheless, melanopsin can trigger G-protein activation in a number of different biochemical assays and cell systems [38, 40, 41, 52], and it seems very likely that it does so in ipRGCs.

If a $G$ protein is in fact the first element downstream of melanopsin in the ipRGC phototransduction cascade, the identity of that $G$ protein is likely to be very informative not only about other probable signaling components but also of the evolutionary origins of ipRGCs. Well-studied phototransduction cascades in the photoreceptors of bilaterian animals fall into one of two broad frameworks $[1,30,48]$. The first, shared by all ciliary photoreceptors (encompassing all vertebrate photoreceptors but ipRGCs), involves $G$ proteins of the $\mathrm{G}_{\mathrm{i} / \mathrm{o}}$ class (including transducin) and cyclic nucleotides as second messengers. The second, characteristic of the rhabdomeric photoreceptors of invertebrate eyes, employs $G$ proteins of the $\mathrm{G}_{\mathrm{q}}$ class and phospholipase C (PLC) as the effector enzyme. As vertebrate photoreceptors that use a rhabdomeric opsin, ipRGCs might conform to either of these canonical cascades, or to neither.

\section{Evidence against a ciliary, cyclic-nucleotide cascade}

The use of $\mathrm{G}_{\mathrm{i} / \mathrm{o}}$ class G-proteins and cyclic nucleotides as second messengers appears highly conserved among ciliary photoreceptors. Such cascades are most familiar from the vertebrate rods and cones, in which photoexcited rhodopsin activates transducin, a $G$ protein belonging to the $G_{i / o}$ class. The liberated alpha subunit of transducin then activates cyclic guanosine monophosphate (cGMP)-specific phosphodiesterase (PDE). The resulting hydrolysis of cGMP reduces the cytosolic concentration of this cyclic nucleotide, closing cGMP-gated channels and attenuating the current that otherwise persists in darkness ("dark current"). The net result of cascade activation is thus membrane hyperpolarization. Other ciliary photoreceptors, such as those of the chicken pineal [28] and lizard parietal eye [56] and even those in invertebrate eyes [10], improvise on this basic theme, but the involvement of $\mathrm{G}_{\mathrm{i} / \mathrm{o}}$ class $\mathrm{G}$ proteins and cGMP appears to be highly conserved.

The only evidence that such a cascade might operate in ipRGCs is that melanopsin can mediate light-dependent activation of rod transducin alpha in a biochemical assay [40]. However, promiscuous interactions of GPCRs with a wide range of $\mathrm{G}$ proteins in biochemical assays are well established, so this capacity sheds little light on the native $\mathrm{G}$ protein in the ipRGC cascade. Indeed, ipRGC photosensitivity appears intact in knockout mice lacking rod transducin alpha [23]. Furthermore, robust ipRGC photo- responses have been recorded in the presence of antagonists of PDE ([63]; Carlson and Berson, unpublished observations). The persistence of ipRGC photosensitivity and melanopsin-mediated visual behaviors in $r d$ mutant mice, e.g., $[43,55,60]$, are also relevant here, as the $r d$ phenotype results from a nonsense mutation in the gene coding for the $\operatorname{PDE} \beta$ subunit [47]. With respect to the ion channel, it is clear from studies in knockout mice that at least the cone cyclic-nucleotide-gated $(\mathrm{CNG})$ channel $(\mathrm{CNG} 3)$ is not required for melanopsin-dependent light responses in ipRGCs [23]. Pharmacological evidence weighs against CNG channels being the light-gated channel in ipRGCs ([63]; Carlson and Berson, unpublished observations). Taken together, there is no compelling evidence that a cyclic-nucleotide-based signaling cascade like that in ciliary photoreceptors mediates ipRGC phototransduction.

\section{Evidence for a rhabdomeric phosphoinositide cascade}

In invertebrate rhabdomeric photoreceptors, phototransduction invariably involves opsin activation of a $G$ protein of the $G_{q / 11}$ family, resulting in the stimulation of PLC $\beta$ and hydrolysis of phosphatidylinositol (4,5)-bisphosphate $\left(\mathrm{PIP}_{2}\right)[1,12,20,29,33,58]$. Less complete information is available about the downstream components of the cascade. Hydrolysis of $\mathrm{PIP}_{2}$ generates the membraneassociated second messenger diacylglycerol (DAG) and the cytosolic inositol 1,4,5 trisphosphate $\left(\mathrm{IP}_{3}\right)$. Both have been implicated to some degree in rhabdomeric phototransduction. In Drosophila ommatidial photoreceptors, $\mathrm{IP}_{3}$ is not essential, and a membrane-delimited cascade involving DAG, polyunsaturated fatty acids, and/or $\mathrm{PIP}_{2}$ itself appear to be playing an essential role [20]. The light-gated channels have been well characterized and represent the original members of the transient receptor potential (TRP) channel proteins, although their gating mechanism remains uncertain. In Limulus ventral photoreceptors, $\mathrm{IP}_{3}$ acting at $\mathrm{IP}_{3}$ receptors to mobilize intracellular $\mathrm{Ca}^{2+}$ may play a significant role in some photoresponse components [12].

In ipRGC phototransduction, although a $\mathrm{G}$ protein is almost certainly an essential component, there is no direct evidence that this belongs to the $\mathrm{G}_{\mathrm{q}}$ class nor that PLC serves as the effector enzyme. Indeed, there is no information available at all concerning which $G$ proteins and PLC isoforms might be expressed in these cells. There is, however, indirect evidence for the plausibility of a $\mathrm{G}_{\mathrm{q}}-\mathrm{PLC}$ cascade in ipRGCs. $\mathrm{G}_{\mathrm{q}}$ has been colocalized with melanopsin in amphioxus rhabdomeric photoreceptors [30]. There is compelling evidence that melanopsin can signal through $\mathrm{G}_{\mathrm{q}}$-class $\mathrm{G}$ proteins and PLC from other cellular systems. In amphibian dermal melanophores, in which melanopsin is the presumptive photopigment, light elevates 
intracellular $\mathrm{IP}_{3}$ levels, and PLC appears to be the effector enzyme for the light-driven pigment dispersion response [27]. Another indirect line of evidence comes from studies of chick RGCs, a small percentage of which express melanopsin [7]. In relatively pure cultures of embryonic chick ganglion cells, transcripts for $\mathrm{G}_{\mathrm{q}}$ but not transducin have been identified [8]. In the same cultures, light suppresses the release of melatonin and this effect, which may be mediated by melanopsin, requires PLC. In two separate heterologous expression systems, mammalian melanopsin signals through a $\mathrm{G}_{\mathrm{q}}$-class $\mathrm{G}$ protein and PLC to generate the transmembrane photocurrent $[41,52]$. Data from a third expression system was at least partly congruent with this picture, implicating a $G$ protein that did not belong to the $\mathrm{G}_{\mathrm{i} / \mathrm{o}}$ class [38]. However, PLC appeared not to be the effector enzyme in this system, making it unlikely that the $\mathrm{G}$ protein in question belongs to the $\mathrm{G}_{\mathrm{q}}$ class. Taken together, the available evidence builds a circumstantial case that melanopsin may be coupled to the light-gated channel in mammalian ipRGCs through a 'rhabdomeric' G-protein cascade. Key evidence on this point is lacking, however. For example, it remains to be shown that ipRGCs express any $\mathrm{G}_{\mathrm{q}}$-class $\mathrm{G}$ proteins or PLC $\beta$. The most glaring evidentiary gap, however, is the absence of data from pharmacological, knockout, or knockdown studies implicating $\mathrm{G}_{\mathrm{q}}$-class $\mathrm{G}$ proteins, PLC, or associated downstream second messengers in ipRGC photoresponses. Our own efforts to block the cascade in intact retinas with antagonists of $\mathrm{G}_{\mathrm{q}}$-class $\mathrm{G}$ proteins and PLC have failed (Carlson and Berson, unpublished). Although this could spell trouble for the hypothesis that a rhabdomeric phosphoinositide cascade is mediating ipRGC light responses, it is possible that such negative results are attributable merely to technical limitations, such as poor access of pharmacological agents to the critical sites of transduction. There is a precedent for such failures in Drosophila photoreceptors, although there is no obvious equivalent in the ipRGCs for the sort of diffusion barriers that are presumably responsible for the negative pharmacological findings in flies.

\section{Identity of the light-gated channel}

The identity of the plasma membrane channels that mediate the ipRGC light response is not known, although recent evidence suggests TRP canonical (TRPC) channels as the most likely candidates ([41, 52, 63], Carlson and Berson, unpublished observations). Light induces a conductance increase in ipRGCs, and the photocurrent reverses near $0 \mathrm{mV}$, suggesting mediation by a nonspecific cationic channel. Ion substitution experiments have suggested that sodium contributes relatively little to the overall current [62], although in our hands, removal of extracellular $\mathrm{Na}^{+}$ substantially alters current-voltage relations [Carlson and Berson, unpublished observations]. In any case, $\mathrm{Ca}^{2+}$ appears to be a significant charge carrier through these channels. This is consistent with the findings of Sekaran et al. [55], who imaged intracellular $\mathrm{Ca}^{2+}$ in retinas largely lacking functional rods and cones. Light triggered a substantial increase in intracellular $\mathrm{Ca}^{2+}$ in a small population of ganglion cells (presumably melanopsin-expressing ipRGCs), and this increase was abolished by the replacement of extracellular $\mathrm{Ca}^{2+}$ with $\mathrm{Cd}^{2+}$ [55]. Although this would seem to support the hypothesis that the light-gated channels have substantial $\mathrm{Ca}^{2+}$ permeability, alternative interpretations are possible. For example, $\mathrm{Ca}^{2+}$ influx through voltage-gated $\mathrm{Ca}^{2+}$ channels (VGCCs) has not been excluded as a source for the recorded $\mathrm{Ca}^{2+}$ signal. VGCCs, which are widely expressed in RGCs, would presumably be activated by the light-evoked depolarization of ipRGCs and would have been blocked by extracellular $\mathrm{Cd}^{2+}$. Further, although transmembrane $\mathrm{Ca}^{2+}$ flux clearly plays an important role, it would be premature to exclude a role for intracellular $\mathrm{Ca}^{2+}$ mobilization because influx of $\mathrm{Ca}^{2+}$ across the plasma membrane can trigger a secondary release of $\mathrm{Ca}^{2+}$ from intracellular stores ('calcium-induced calcium release') in many cells. This would be in addition to any $\mathrm{Ca}^{2+}$ mobilization that might occur as a result of PLC activation and consequent liberation of $\mathrm{Ca}^{2+}$ from $\mathrm{IP}_{3}$-sensitive stores [32, 52].

The similarity of both the opsin and the presumptive signaling cascade in ipRGCs to those in rhabdomeric photoreceptors has encouraged speculation that the lightgated channel might belong to the TRP family. Originally identified in Drosophila rhabdomeric photoreceptors [39], these channel proteins are now recognized as forming a large family of tetrameric, calcium-permeable channels involved in a variety of functions ranging from sensory transduction to intracellular calcium homeostasis [53]. Of particular relevance to ipRGCs are the vertebrate TRP proteins of the TRPC family, as these bear the greatest resemblance to Drosophila TRP including in their gating by activation of GPCRs coupled to phosphoinositide cascades. Indeed, the current-voltage relations of the ipRGC photocurrent ([62]; Carlson and Berson, unpublished observations) resemble those of TRPC-mediated currents in some heterologous expression systems [25, 26, 53], with reversal near $0 \mathrm{mV}$ and both inward and outward rectification. TRPC proteins form nonspecific cationic channels with substantial $\mathrm{Ca}^{2+}$ permeability, matching known features of the ipRGC light-activated channel. Pharmacological agents known to effectively block TRPC channels attenuate or abolish the ipRGC photocurrent ([63]; Carlson and Berson, unpublished observations), but none of these agents is specific for TRPC channels. In heterologous expression systems, melanopsin has been shown to be able to mediate light-triggered gating of TRPC channels [41, 52]. At least 
one TRPC protein (TRPC6) has been detected in ipRGCs by immunohistochemistry [63]. Thus, there is ample evidence to view TRPC channels as good candidates for the light-gated channel in ipRGCs. A stronger case awaits better pharmacological tools or evidence from genetic manipulation experiments that the expression of these channels is necessary for the ipRGC photoresponse. It will also be important to catalog the full range of TRPC proteins expressed in ipRGCs in view of the potential for formation of heteromultimeric channels [53].

\section{The road ahead}

Although substantial progress has been made toward identifying the phototransduction cascade in mammalian ganglion-cell photoreceptors, there are many glaring gaps in our understanding. The evidence that melanopsin forms the photopigment in these cells is overwhelming, although much remains to be understood about the specifics of its behavior under physiological conditions in ipRGCs. Of particular importance will be to establish the identity of its chromophore and to understand the relative contributions of intrinsic photoreversal and extrinsic enzymatic retinoid cycles in the regeneration of photoexcitable pigment. Also largely unexplored are mechanisms for response termination and adaptation, melanopsin's selectivity of interaction with a range of G-protein partners, and the identity of its intracellular residues contributing to those interactions.

As for downstream signaling, there is clearly mounting evidence for the involvement of a phosphoinositide signaling cascade resembling that in rhabdomeric photoreceptors. There may be a particularly close resemblance to the cascade worked out in Drosophila photoreceptors, inasmuch as the TRPC channels are emerging as the best candidates for the light-activated channels. However, there remain troubling gaps in the empirical foundation for this view, especially the inability so far to demonstrate that $\mathrm{G}_{\mathrm{q}}$-class $\mathrm{G}$ proteins or PLC are essential for ipRGC photoresponses. One likely barrier to obtaining such evidence is the difficulty of exerting sufficient pharmacological control over cells with transduction machinery localized in part to very fine dendritic processes deeply embedded in the retinal tissue. An important challenge for future work, then, is to develop methods for recording photoresponses from dissociated ipRGCs. Ultimately, obtaining definitive evidence on the nature of the cascade will probably require experimental manipulation of critical genes, either in mouse models or transfection studies.

One of the most exciting aspects of the emerging picture of the ipRGC phototransduction cascade is that it provides a critical test of the emerging hypothesis that ipRGCs and invertebrate rhabdomeric photoreceptors share a common evolutionary origin $[1,48]$. If this proves to be true, it will be of interest to pursue comparative studies to learn which aspects of the ipRGC response to light are inherent in the highly conserved signaling cascade on which their photosensitivity is based and which are adaptations to their particular functional roles in mammalian vision.

\section{References}

1. Arendt D (2003) Evolution of eyes and photoreceptor cell types. Int J Dev Biol 47:563-571

2. Belenky MA, Smeraski CA, Provencio I, Sollars PJ, Pickard GE (2003) Melanopsin retinal ganglion cells receive bipolar and amacrine cell synapses. J Comp Neurol 460:380-393

3. Bellingham J, Foster RG (2002) Opsins and mammalian photoentrainment. Cell Tissue Res 309:57-71

4. Berson DM (2003) Strange vision: ganglion cells as circadian photoreceptors. Trends Neurosci 26:314-320

5. Berson DM, Dunn FA, Takao M (2002) Phototransduction by retinal ganglion cells that set the circadian clock. Science 295:1070-1073

6. Brown RL, Robinson PR (2004) Melanopsin-shedding light on the elusive circadian photopigment. Chronobiol Int 21:189-204

7. Chaurasia SS, Rollag MD, Jiang G, Hayes WP, Haque R et al (2005) Molecular cloning, localization and circadian expression of chicken melanopsin (Opn4): differential regulation of expression in pineal and retinal cell types. J Neurochem 92:158-170

8. Contin MA, Verra DM, Guido ME (2006) An invertebrate-like phototransduction cascade mediates light detection in the chicken retinal ganglion cells. FASEB J 20:2648-2650

9. Dacey DM, Liao HW, Peterson BB, Robinson FR, Smith VC et al (2005) Melanopsin-expressing ganglion cells in primate retina signal colour and irradiance and project to the LGN. Nature 433:749-754

10. del Pilar Gomez M, Nasi E (1995) Activation of light-dependent $\mathrm{K}+$ channels in ciliary invertebrate photoreceptors involves cGMP but not the IP3/Ca2+ cascade. Neuron 15:607-618

11. Dkhissi-Benyahya O, Rieux C, Hut RA, Cooper HM (2006) Immunohistochemical evidence of a melanopsin cone in human retina. Invest Ophthalmol Vis Sci 47:1636-1641

12. Dorlochter M, Stieve H (1997) The Limulus ventral photoreceptor: light response and the role of calcium in a classic preparation. Prog Neurobiol 53:451-515

13. Doyle SE, Castrucci AM, McCall M, Provencio I, Menaker M (2006) Nonvisual light responses in the Rpe65 knockout mouse: rod loss restores sensitivity to the melanopsin system. Proc Natl Acad Sci USA 103:10432-10437

14. Foster RG (2002) Keeping an eye on the time: the Cogan Lecture. Invest Ophthalmol Vis Sci 43:1286-1298

15. Freedman MS, Lucas RJ, Soni B, von Schantz M, Munoz M et al (1999) Regulation of mammalian circadian behavior by non-rod, noncone, ocular photoreceptors [see comments]. Science 284:502-504

16. Fu Y, Liao HW, Do MT, Yau KW (2005) Non-image-forming ocular photoreception in vertebrates. Curr Opin Neurobiol 15:415-422

17. Fu Y, Zhong H, Wang MH, Luo DG, Liao HW et al (2005) Intrinsically photosensitive retinal ganglion cells detect light with a vitamin A-based photopigment, melanopsin. Proc Natl Acad Sci USA 102:10339-10344

18. Gooley JJ, Lu J, Chou TC, Scammell TE, Saper CB (2001) Melanopsin in cells of origin of the retinohypothalamic tract. Nat Neurosci 4:1165

19. Hannibal J, Hindersson P, Knudsen SM, Georg B, Fahrenkrug J (2002) The photopigment melanopsin is exclusively present in pituitary adenylate cyclase-activating polypeptide-containing retinal ganglion cells of the retinohypothalamic tract. J Neurosci 22:RC191 
20. Hardie RC, Raghu P (2001) Visual transduction in Drosophila. Nature 413:186-193

21. Hattar S, Kumar M, Park A, Tong P, Tung J et al (2006) Central projections of melanopsin-expressing retinal ganglion cells in the mouse. J Comp Neurol 497:326-349

22. Hattar S, Liao HW, Takao M, Berson DM, Yau KW (2002) Melanopsin-containing retinal ganglion cells: architecture, projections, and intrinsic photosensitivity. Science 295:1065-1070

23. Hattar S, Lucas RJ, Mrosovsky N, Thompson S, Douglas RH et al (2003) Melanopsin and rod-cone photoreceptive systems account for all major accessory visual functions in mice. Nature 424:76-81

24. Hillman P, Hochstein S, Minke B (1983) Transduction in invertebrate photoreceptors: role of pigment bistability. Physiol Rev 63:668-772

25. Hofmann T, Obukhov AG, Schaefer M, Harteneck C, Gudermann $\mathrm{T}$ et al (1999) Direct activation of human TRPC6 and TRPC3 channels by diacylglycerol. Nature 397:259-263

26. Inoue R, Okada T, Onoue H, Hara Y, Shimizu S et al (2001) The transient receptor potential protein homologue TRP6 is the essential component of vascular alpha(1)-adrenoceptor-activated $\mathrm{Ca}(2+)$-permeable cation channel. Circ Res 88:325-332

27. Isoldi MC, Rollag MD, Castrucci AM, Provencio I (2005) Rhabdomeric phototransduction initiated by the vertebrate photopigment melanopsin. Proc Natl Acad Sci USA 102:1217-1221

28. Kasahara T, Okano T, Yoshikawa T, Yamazaki K, Fukada Y (2000) Rod-type transducin alpha-subunit mediates a phototransduction pathway in the chicken pineal gland. J Neurochem 75:217-224

29. Kikkawa S, Tominaga K, Nakagawa M, Iwasa T, Tsuda M (1996) Simple purification and functional reconstitution of octopus photoreceptor Gq, which couples rhodopsin to phospholipase C. Biochemistry 35:15857-15864

30. Koyanagi M, Kubokawa K, Tsukamoto H, Shichida Y, Terakita A (2005) Cephalochordate melanopsin: evolutionary linkage between invertebrate visual cells and vertebrate photosensitive retinal ganglion cells. Curr Biol 15:1065-1069

31. Kumbalasiri T, Provencio I (2005) Melanopsin and other novel mammalian opsins. Exp Eye Res 81:368-375

32. Kumbalasiri T, Rollag MD, Isoldi $M$, de Lauro Castrucci AM, Provencio I (2007) Melanopsin triggers the release of internal calcium stores in response to light. Photochem Photobiol (in press) DOI 10.1562/2006-07-11-RA-964

33. Lee YJ, Shah S, Suzuki E, Zars T, O’Day PM et al (1994) The Drosophila dgq gene encodes a $\mathrm{G}$ alpha protein that mediates phototransduction. Neuron 13:1143-1157

34. Lucas RJ (2006) Chromophore regeneration: melanopsin does its own thing. Proc Natl Acad Sci USA 103:10153-10154

35. Lucas RJ, Douglas RH, Foster RG (2001) Characterization of an ocular photopigment capable of driving pupillary constriction in mice. Nat Neurosci 4:621-626

36. Lucas RJ, Freedman MS, Munoz M, Garcia-Fernandez JM, Foster RG (1999) Regulation of the mammalian pineal by non-rod, noncone, ocular photoreceptors [see comments]. Science 284:505-507

37. Lucas RJ, Hattar S, Takao M, Berson DM, Foster RG et al (2003) Diminished pupillary light reflex at high irradiances in melanopsinknockout mice. Science 299:245-247

38. Melyan Z, Tarttelin EE, Bellingham J, Lucas RJ, Hankins MW (2005) Addition of human melanopsin renders mammalian cells photoresponsive. Nature 433:741-745

39. Montell C (2005) TRP channels in Drosophila photoreceptor cells. J Physiol 567:45-51

40. Newman LA, Walker MT, Brown RL, Cronin TW, Robinson PR (2003) Melanopsin forms a functional short-wavelength photopigment. Biochemistry 42:12734-12738

41. Panda S, Nayak SK, Campo B, Walker JR, Hogenesch JB et al (2005) Illumination of the melanopsin signaling pathway. Science 307:600-604
42. Panda S, Provencio I, Tu DC, Pires SS, Rollag MD et al (2003) Melanopsin is required for non-image-forming photic responses in blind mice. Science 301:525-527

43. Panda S, Sato TK, Castrucci AM, Rollag MD, DeGrip WJ et al (2002) Melanopsin (Opn4) requirement for normal light-induced circadian phase shifting. Science 298:2213-2216

44. Peirson S, Foster RG (2006) Melanopsin: another way of signaling light. Neuron 49:331-339

45. Peirson SN, Bovee-Geurts PH, Lupi D, Jeffery G, DeGrip WJ et al (2004) Expression of the candidate circadian photopigment melanopsin (Opn4) in the mouse retinal pigment epithelium. Brain Res Mol Brain Res 123:132-135

46. Perez-Leon JA, Warren EJ, Allen CN, Robinson DW, Lane Brown $\mathrm{R}$ (2006) Synaptic inputs to retinal ganglion cells that set the circadian clock. Eur J Neurosci 24:1117-1123

47. Pittler SJ, Baehr W (1991) Identification of a nonsense mutation in the rod photoreceptor cGMP phosphodiesterase beta-subunit gene of the rd mouse. Proc Natl Acad Sci USA 88:8322-8326

48. Plachetzki DC, Serb JM, Oakley TH (2005) New insights into the evolutionary history of photoreceptor cells. Trends Ecol Evol 20:465-467

49. Provencio I, Jiang G, De Grip WJ, Hayes WP, Rollag MD (1998) Melanopsin: an opsin in melanophores, brain, and eye. Proc Natl Acad Sci USA 95:340-345

50. Provencio I, Rodriguez IR, Jiang G, Hayes WP, Moreira EF et al (2000) A novel human opsin in the inner retina. J Neurosci 20:600605

51. Provencio I, Rollag MD, Castrucci AM (2002) Photoreceptive net in the mammalian retina. This mesh of cells may explain how some blind mice can still tell day from night. Nature 415:493

52. Qiu X, Kumbalasiri T, Carlson SM, Wong KY, Krishna V et al (2005) Induction of photosensitivity by heterologous expression of melanopsin. Nature 433:745-749

53. Ramsey IS, Delling M, Clapham DE (2006) An introduction to TRP channels. Annu Rev Physiol 68:619-647

54. Rollag MD, Berson DM, Provencio I (2003) Melanopsin, ganglion-cell photoreceptors, and mammalian photoentrainment. J Biol Rhythms 18:227-234

55. Sekaran S, Foster RG, Lucas RJ, Hankins MW (2003) Calcium imaging reveals a network of intrinsically light-sensitive innerretinal neurons. Curr Biol 13:1290-1298

56. Su CY, Luo DG, Terakita A, Shichida Y, Liao HW et al (2006) Parietal-eye phototransduction components and their potential evolutionary implications. Science 311:1617-1621

57. Terakita A (2005) The opsins. Genome Biol 6:213

58. Terakita A, Hariyama T, Tsukahara Y, Katsukura Y, Tashiro H (1993) Interaction of GTP-binding protein Gq with photoactivated rhodopsin in the photoreceptor membranes of crayfish. FEBS Lett 330:197-200

59. Tu DC, Owens LA, Anderson L, Golczak M, Doyle SE et al (2006) Inner retinal photoreception independent of the visual retinoid cycle. Proc Natl Acad Sci USA 103:10426-10431

60. Tu DC, Zhang D, Demas J, Slutsky EB, Provencio I et al (2005) Physiologic diversity and development of intrinsically photosensitive retinal ganglion cells. Neuron 48:987-999

61. Van Gelder RN (2005) Nonvisual ocular photoreception in the mammal. Methods Enzymol 393:746-755

62. Warren EJ, Allen CN, Brown RL, Robinson DW (2003) Intrinsic light responses of retinal ganglion cells projecting to the circadian system. Eur J Neurosci 17:1727-1735

63. Warren EJ, Allen CN, Brown RL, Robinson DW (2006) The light-activated signaling pathway in SCN-projecting rat retinal ganglion cells. Eur J Neurosci 23:2477-2487

64. Wong KY, Dunn FA, Berson DM (2005) Photoreceptor adaptation in intrinsically photosensitive retinal ganglion cells. Neuron 48:1001-1010 